\title{
Mining Large Query Induced Graphs towards a Hierarchical Query Folksonomy
}

\author{
Alexandre P. Francisco ${ }^{1, \star}$, Ricardo Baeza-Yates ${ }^{2}$, and Arlindo L. Oliveira ${ }^{1}$ \\ 1 INESC-ID / CSE Dept, IST, Tech Univ of Lisbon, Portugal \\ ${ }^{2}$ Yahoo! Research Barcelona, Spain \& Santiago, Chile
}

\begin{abstract}
The human interaction through the web generates both implicit and explicit knowledge. An example of an implicit contribution is searching, as people contribute with their knowledge by clicking on retrieved documents. Thus, an important and interesting challenge is to extract semantic relations among queries and their terms from query logs. In this paper we present and discuss results on mining large query log induced graphs, and how they contribute to query classification and to understand user intent and interest. Our approach consists on efficiently obtaining a hierarchical clustering for such graphs and, then, a hierarchical query folksonomy. Results obtained with real data provide interesting insights on semantic relations among queries and are compared with conventional taxonomies, namely the ODP categorization.
\end{abstract}

\section{Introduction}

Nowadays the Web is the biggest representation of human knowledge, where people contribute with content either explicitly or implicitly. An example of an implicit contribution is searching, as people contribute with their knowledge by clicking on retrieved documents. Thus, queries submitted to search engines carry implicit knowledge and they can be seen as equivalent to tags associated to clicked documents. An interesting challenge is then to extract relevant semantic relations from query logs, which have several interesting applications. For instance, ranking algorithms, query recommendation systems and advertisement systems integrate such semantic information to improve their results.

In this paper we discuss query classification and meaning, and not URL tagging and folksonomies. We use click-data to infer relationships and similarities among queries. Then, by finding closely related queries, we are able to define a hierarchical query folksonomy associating tags to queries. Note that this approach may associate a tag to a query even if that tag is not part of the query, leading to query contextualization. Our approach relies on graphs to represent relations among queries and on efficient graph mining techniques to uncover relations. According to SearchEngineWatch.com, the number of queries per day is of the order of hundreds of millions, leading to huge query graphs. On the other hand, the number of potential relations and applications is also huge.

\footnotetext{
* Work done while visiting Yahoo! Research Barcelona. Email: aplf@ist.utl.pt

E. Chavez and S. Lonardi (Eds.): SPIRE 2010, LNCS 6393, pp. 238-243 2010.

(C) Springer-Verlag Berlin Heidelberg 2010
} 
Our study follows recent works on the analysis of query graphs [310, which introduce the notion of click induced graph and present several results concerning semantic relations among queries. Here we propose three main contributions: a new heuristic to detect and remove noisy relations among queries mostly caused by multi-topical URLs; an efficient hierarchical clustering method for weighted graphs to extract semantic relations from query graphs; and, given a hierarchical clustering, a method to infer a query folksonomy and semantic relations among queries. We use a sample of a query log of the Yahoo! search engine to evaluate our approach and we compare our results with a query classification obtained by mapping queries over the Open Directory Project (ODP) categories.

\section{Related Work}

Most of the work on query similarity is related to query expansion or query clustering, common tasks in many applications such as query recommendation systems. Wen et al [15] proposed to cluster similar queries using four notions of query distance: (1) based on keywords or phrases of the query; (2) based on string matching of keywords; (3) based on common clicked URLs; and (4) based on the distance of the clicked documents in some predefined hierarchy. As the average number of words in queries is small and the number of clicks in the answer pages is also small [1, notions (1) and (2) generate distance matrices that are very sparse. For notion (4) we need a concept taxonomy and the clicked documents must be classified into that taxonomy as well, something that usually requires direct human intervention and that cannot be done in a large scale. Although notion (3) can generate also sparse distance matrices, the sparsity can be greatly reduced by using large query logs. Previous works have used notion (3) [4], or even variants combining (1) and (3) [16.

Baeza-Yates et al. 2] used the content of clicked Web pages to define a termweight vector model for a query. In their work each term in a clicked URL is weighted according to the number of occurrences of the query and the number of clicks of the documents in which the term appears. Then, the similarity of two queries is given by the cosine similarity of their vector representations. This notion of query similarity is based on common clicked URLs as (3) and has several advantages. It is simple and easy to compute and makes it possible to relate queries that happen to be worded differently but stem from the same topic. More recently, Shen et al. [13] also used the notion (3) to cluster similar queries and build a query taxonomy. They also consider the terms in the clicked documents instead of the terms in the queries. In this paper we represent queries in a high dimensional space and we use also the cosine similarity, but each dimension corresponds to a URL. This notion uses common clicked URLs and it was introduced by Baeza-Yates and Tiberi 3 .

Chuang et al. 5617] also used query logs to build a query taxonomy to also cluster answers. However they do not use any user feedback, like user clicks. Moreover, this is not the same as building a taxonomy of the queries, which is what we call a query taxonomy or folksonomy. 

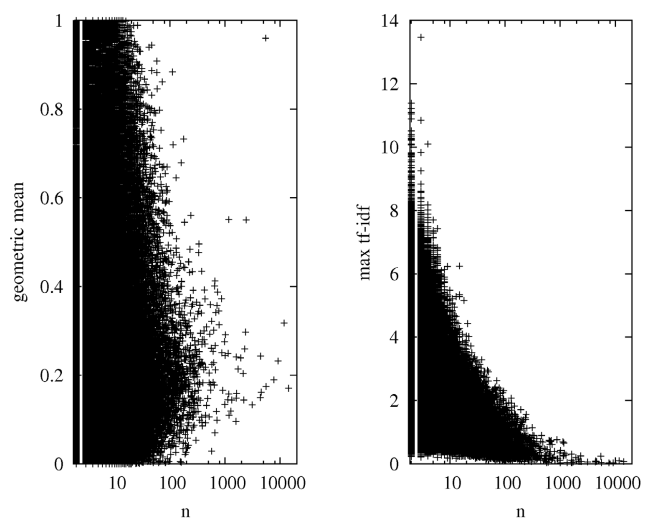

Fig. 1. On left, URL weight contribution geometric mean versus URL coverage size $n$. On right, given the set of terms associated to the queries covered by each URL, URL maximum tf-idf score versus URL coverage size $n$.

\section{Click Induced Graph}

Given a query $q$, the cover $\mu(q)$ of $q$ is the set of URLs clicked by $q$. The click induced graph $G=(V, E)$ is an undirected graph with queries as nodes, $V=\mathcal{Q}$, and where $\left(q_{1}, q_{2}\right) \in E$ whenever $q_{1}$ and $q_{2}$ share at least one common clicked URL, $\mu\left(q_{1}\right) \cap \mu\left(q_{2}\right) \neq \emptyset$. Edges are weighted according to the cosine similarity $\sigma$ of the queries they connect, with each query $q$ being represented in a high dimensional space. Each dimension corresponds to a unique URL $u$ and the weight is the frequency ratio with which the URL $u$ was clicked for the query $q$.

In this paper we considered a query log piece from the Yahoo! search engine. The data was collected in April 2005 and contains 2,822,337 queries with at least one clicked URL and 4,927,980 different URLs. From these, only 660,910 URLs were clicked for at least one query. On average, each query has 2.39 distinct clicks and each URL is clicked by 1.37 distinct queries. Click distributions, per query and per URL, follow a power law with exponents 3.50 and 2.59 , respectively.

The main purpose of the click induced graph is to represent semantic relations between queries and to enable knowledge extraction. But, for the studied query log, we have that about $75 \%$ and $50 \%$ of edges have weights bellow 0.5 and 0.273 , respectively. Thus, there are many connected queries which are not much similar. Such noisy relations are mostly due to URLs covering dubious topics, several topics or very general topics, i.e., multi-topical URLs.

An approach to remove noise is to ignore contributions from multi-topical URLs. Baeza-Yates and Tiberi [3] suggested that such URLs are the ones that contribute more to edges with low weights. But, we observed that URLs which contribute more to low weighted edges also may contribute more to high weighted edges. In fact there is a positive correlation between the number of queries covered by a URL and its contribution to edge weights (see Fig. 1). We tackle this problem by considering as documents the terms among the queries covered by 
each URL and by computing the tf-idf score for each term as usual. We observed that multi-topical URLs have a low average tf-idf score, even when we select only high related queries for which those URLs were clicked. Hence, we propose to compute the maximum tf-idf among the bag of terms associated to each URL and select the URLs with lowest score as multi-topical candidates. In Fig. 1 we have the maximum tf-idf score against URL coverage size for the query log analyzed. In what follows, we ignore $0.05 \%$ of the URLs with lowest score, filtering the click induced graph in a conservative way. Note that many of the selected URLs have a large coverage and, maybe unexpectedly, they are not spam URLs.

The resulting click induced graph has 23,177,430 edges, about $6.44 \%$ of the size of the full click induced graph. Since we continue having low weighted edges, we remove $10 \%$ of the edges with lowest score, which have weights lower than 0.043 . The filtered click induced graph has 20,974,257 edges and 1,648,649 connected components. The giant component and the second largest component have 861,903 and 64 vertices, respectively. There are now 1,474,249 singleton vertices. The degree distribution follows a power law with exponent 1.65. Our approach to remove noise and multi-topical URLs dramatically reduces the size of the click induced graph, keeping its structure almost unchanged.

\section{Graph Clustering and Induced Query Folksonomy}

One of the hardest problems in graph mining is finding graph community structure or graph clustering. Usually, clusters are groups of vertices such that the number of edges within them is higher than the number of edges among different groups. This problem has recently attracted a large interest [9]. In this paper we follow a two stage approach. We find a set of seed sets and, then, we apply a local optimization method. This approach is related to methods based on global partition and local expansion 1214, but avoiding traditional global partitioning. Since we are interested in forming clusters of similar queries, our approach consists of joining similar queries, i.e., we define cores based on vertex structure similarity. Let $G=(V, E)$ be a graph and $\sigma: E \longrightarrow \mathbb{R}_{0}^{+}$the edge weight function. Given two connected vertices $\left(v_{1}, v_{2}\right) \in E$, their structural similarity $\eta$ takes values in $[0,1]$ and is given by

$$
\frac{\sum_{w \in N_{12}} \sigma\left(v_{1}, w\right)+\sigma\left(v_{2}, w\right)}{\left|N_{12}\right|} \frac{2 \sigma\left(v_{1}, v_{2}\right)+\sum_{w \in N_{12}} \sigma\left(v_{1}, w\right) \sigma\left(v_{2}, w\right)}{\sqrt{1+\sum_{w \in N_{1}} \sigma\left(v_{1}, w\right)^{2}} \sqrt{1+\sum_{w \in N_{2}} \sigma\left(v_{2}, w\right)^{2}}},
$$

where $N_{1}$ and $N_{2}$ are the sets of neighbors of $v_{1}$ and $v_{2}$, respectively, and $N_{12}=N_{1} \cap N_{2}$. The first term, the weight mean among common neighbors, was introduced because the second term, a cosine similarity based score, takes value 1.0 whenever the vertices $v_{1}$ and $v_{2}$ share all neighbors, even if they are connected through edges with low weights. Given $\varepsilon>0, C \subseteq V$ is a core if $C$ is a connected component composed only of edges with weights higher than $\varepsilon$. We can enumerate the set of cores in a graph for different values of $\varepsilon>0$ and, by considering the edges in decreasing order of $\eta$, we obtain a hierarchy of cores. 
Then, we apply the local partition method proposed by Chung [8] to each core, which expands it minimizing the conductance score $\Phi$.

The induced query taxonomy is obtained by associating the most relevant terms to each node in the hierarchical clustering tree. We compute the set of terms associated to each node by grouping together all queries in the underlying subtree and by inspecting the queries. Those sets of terms become our documents and we infer the most relevant terms for each node by computing the tf-idf score for each term. Such terms become the tags for that node. Since click induced graphs are scale-free and have a giant component, we may want to define a threshold on the tf-idf score. Internal nodes corresponding to the giant component, or even to part of it, have usually bad quality tags which do not bring relevant semantic information.

\section{Experimental Evaluation}

For lack of space, we summarize briefly our results for the click induced graph described above. Full results are available in a technical report [11]. By considering different values for $\varepsilon$, we saw that the method effectively clusters the graph. For instance, with $\varepsilon=0.4$, the biggest cluster is much smaller, about $1.1 \%$ of the original giant component. We note also that values for average conductance $\Phi$ are less than 0.1. Nevertheless, we obtain many small clusters for any cut of the hierarchical clustering tree, corresponding to loosely connected clusters that could appear connected if we consider larger query logs. Many are composed of highly specific queries or navigational queries, for which the search engine may return a low number of results and where the user clearly knows what he wants.

As mentioned before, the local optimization may lead to overlaps among clusters, providing interesting information with respect to query ambiguity, context, topic and polysemy. Terms like "windows" and "wine" are examples of polysemic terms that appear within overlaps of rather different clusters. Thus, an approach to identify term polysemy is to compare the bag of terms among overlapping clusters and, if a query is in two clusters but they share few terms, then the query shall be polysemic. Similarly, by analyzing similar words in the same cluster we can detect misspellings.

Given the hierarchical clustering described above, we build the induced query folksonomy for which tf-idf scores become meaningful only for $\varepsilon>0.3$, i.e., when the giant component vanishes. For $\varepsilon \leq 0.3$, the tf-idf score for the giant component takes values between 0.05 and 0.07 , and the most relevant term is "free". Evaluating the query classification is difficult since it is very different from traditional directories. Here we try to compare it with the ODP in order to understand how different are these two ways of expressing knowledge. We mapped all queries over the ODP categories, obtaining several category paths for each query. Then we compare the ODP paths with the induced folksonomy. Since folksonomy labels are not comparable to the categories in the ODP mapping, they are not topic based, we evaluate the clusters by comparing the common ODP path prefix among the queries. Given two queries $q_{1}$ and $q_{2}$, we select 
the two most similar ODP category paths $p_{1}$ and $p_{2}$, i.e., the ones which share the longest common prefix $\pi\left(p_{1}, p_{2}\right)$. Then we compute the score $\sigma_{\text {odp }}\left(p_{1}, p_{2}\right)=$ $\left|\pi\left(p_{1}, p_{2}\right)\right| / \max \left\{\left|p_{1}\right|,\left|p_{2}\right|\right\}$, where $|\cdot|$ denotes the path length. The ODP score for a given cluster is the average of the score $\sigma_{\text {odp }}$ for all pairs of queries in that cluster. For all snapshots of the hierarchical clustering for different values of $\varepsilon$, more than $50 \%$ of the clusters have an ODP score higher than 0.5 and $30 \%$ to $39 \%$ of them have an ODP score equal to 1.0. For $\varepsilon>0.3$, after we cluster the giant component, the ODP score increases with the hierarchical clustering depth, revealing that clusters at higher depths have better quality. This is also supported by the tf-idf scores.

\section{References}

1. Baeza-Yates, R.: Applications of web query mining. In: Losada, D.E., FernándezLuna, J.M. (eds.) ECIR 2005. LNCS, vol. 3408, pp. 7-22. Springer, Heidelberg (2005)

2. Baeza-Yates, R., Hurtado, C., Mendoza, M.: Query clustering for boosting web page ranking. In: Favela, J., Menasalvas, E., Chávez, E. (eds.) AWIC 2004. LNCS (LNAI), vol. 3034, pp. 164-175. Springer, Heidelberg (2004)

3. Baeza-Yates, R.A., Tiberi, A.: Extracting semantic relations from query logs. In: SIGKDD, pp. 76-85. ACM, New York (2007)

4. Beeferman, D., Berger, A.: Agglomerative clustering of a search engine query log. In: SIGKDD. ACM, New York (1999)

5. Chuang, S.L., Chien, L.F.: Towards automatic generation of query taxonomy: A hierarchical query clustering approach. In: IEEE International Conference on Data Mining. IEEE, Los Alamitos (2002)

6. Chuang, S.L., Chien., L.F.: Automatic query taxonomy generation for information retrieval applications. Online Information Review 27(5) (2003)

7. Chuang, S.L., Chien, L.F.: Enriching web taxonomies through subject categorization of query terms from search engine logs. Decision Support System 30(1) (2003)

8. Chung, F.: The heat kernel as the pagerank of a graph. Proceedings of the National Academy of Sciences 104(50), 19735 (2007)

9. Fortunato, S.: Community detection in graphs. Physics Reports 486, 75-174 (2010)

10. Francisco, A.P., Baeza-Yates, R., Oliveira, A.L.: Clique analysis of query log graphs. In: Amir, A., Turpin, A., Moffat, A. (eds.) SPIRE 2008. LNCS, vol. 5280, pp. 188199. Springer, Heidelberg (2008)

11. Francisco, A.P., Baeza-Yates, R., Oliveira, A.L.: Mining query logs induced graphs. Tech. Rep.??/2010, INESC-ID (2010)

12. Leskovec, J., Lang, K.J., Dasgupta, A., Mahoney, M.W.: Community structure in large networks: Natural cluster sizes and the absence of large well-define clusters. arXiv:0810.1355 (2008)

13. Shen, D., Qin, M., Chen, W., Yang, Q., Chen, Z.: Mining Web Query Hierarchies from Clickthrough Data. In: AAAI 2007, pp. 341-346. AAAI Press, Menlo Park (2007)

14. Wei, F., Qian, W., Wang, C., Zhou, A.: Detecting Overlapping Community Structures in Networks. World Wide Web 12(2), 235-261 (2009)

15. Wen, J., Mie, J., Zhang, H.: Clustering user queries of a search engine. In: Proc. of the 10th International World Wide Web Conference. W3C (2001)

16. Zaiane, O.R., Strilets, A.: Finding similar queries to satisfy searches based on query traces. In: Efficient Web-Based Information Systems (EWIS) (2002) 